\title{
Multi-Agent Rigid Formations: A Study of Robustness to the Loss of Multiple agents
}

\author{
S. Alireza Motevallian, Changbin Yu, Brian D.O. Anderson
}

\begin{abstract}
In this paper we study the robustness of information architectures to control a formation of autonomous agents. If agents are expected to work in hazardous environments like battle-fields, the formations are prone to multiple agent/link loss. Due to the higher severity of agent loss than link loss, the main contribution of this paper is to propose information architectures for shape-controlled multi-agent formations, which are robust against the loss of multiple agents. A formation is said to be rigid if by actively maintaining a designated set of inter-agent distances, the formation preserves its shape. We will use the rigidity theory to formalize the robust architecture problem. In particular we study the properties of formation graphs which remain rigid after the loss of any set of up to $k-1$ vertices. Such a graph is called $k$-vertex rigid. We provide a set of distinct necessary and sufficient conditions for these graphs. We then show that 3-vertex rigidity is the highest possible robustness one can achieve by just adding a small number of edges to a minimally rigid graph, i.e. retention of rigidity given the loss of 3 or more agents of a formation requires many more inter-agent distances to be specified than when maintaining rigidity with no, one or two agent losses. Based on this result, we further focus on 3-vertex rigid graphs and characterize a class of information architectures (with minimum number of control links) which are robust against the loss of up to two agents.
\end{abstract}

Index Terms-Formation Control, Robustness, Rigidity, Redundant Rigidity

\section{INTRODUCTION}

Recently, autonomous agents and specifically UAVs (unmanned aerial vehicles) have found significant interests among researchers. UAVs have become an enabling technology in military application such as surveillance and reconnaissance over several decades [4], [12]. Today, there is also an increasing interest in UAVs for civil application such as environmental monitoring and exploration [1], [7]. In many applications it is desirable to have several autonomous UAVs flying in a formation [7]. The formation control task is to control, normally in a distributed manner, a set of inter-agent distances such that a prescribed shape is achieved and the formation moves as a cohesive whole [1], [5]. The reason is that when these agents, engaged in surveillance or exploration missions, move in a formation with a specific shape, they usually synthesize an antenna of magnitude far larger than a single agent [1], [14]. For certain distributed antenna shapes, this can lead to better sensitivity in target detection or localization over the area of interest.

Many of these missions for UAVs enforce the existence of a high level of autonomy of the vehicles, specifically in hazardous

This work is supported by USAF-AOARD-10-4102. S. Alireza Motevallian is supported by National ICT Australia (NICTA). The work of C. Yu was supported by the Australian Research Council through a Queen Elizabeth II Fellowship, Discovery Project DP-110100538 and Overseas Expert Program of Shandong Province. Brian D.O. Anderson is supported by the ARC and NICTA.

S. Alireza Motevallian and Brian D.O. Anderson are with the Research School of Information Sciences and Engineering, the Australian National University and National ICT Australia.

C. Yu is with the Australian National University and NICTA Ltd, Canberra, A.C.T., Australia, and also with Shandong Computer Science Center, Jinan, China.

Emails: \{alireza.motevallian,brad.yu, brian.anderson\}@anu.edu.au.

Correspondence to: S. Alireza Motevallian, RSISE building 115, The Australian National University, Canberra, ACT, 0200, Australia. Tel: +61 2 6125 8639. Fax: +61261258660. environments or in the presence of communication blackouts [9]. This autonomy can provide higher precision, fault tolerance and reliability in the mission accomplishment.

In hazardous environments such as battle-fields, the robustness of the formation is of high importance [9], [4]. Robustness here, means ensuring a successful accomplishment of the mission in different operational scenarios, when both some agents and communication links may be lost due to mechanical failure, enemy attack, jamming, an agent leaves the formation deliberately, etc.

In [4], as cited in [17], some vulnerability issues of UAVs are studied based on the actual records of the past and the potential issues are mainly classified as

- communication link loss as a result of jamming or occlusion

- enemy attack of one or more UAVs in the formation

- loss of an agent or communication link as a result of a mechanical or electrical failure, without enemy attack but possibly due to environmental changes (heat, wind, etc.).

Based on the above study the authors concluded the necessity of reducing the vulnerability of UAV to such threats.

It is evident that the robustness of the formation to an agent loss demands more than robustness to a single link loss, as the loss of one agent implies the loss of all control links incident to it. Therefore, we focus on the multiple agent loss problem throughout this paper. The issue can be dealt with in many different ways [15]. However, in this paper we incorporate a proactive approach. We introduce the robustness into the information architecture a priori , by using redundant links, in order to mitigate the effect of multiple agent loss. The measure of robustness here, is the number of agents the formation can afford to lose while preserving its cohesiveness.

We assume a graphical abstraction of the multi-agent formation, via an undirected graph $G=(V, E)$, in which each vertex corresponds to an agent and an edge corresponds to a bidirectional distance control law, actively maintaining the distance between the corresponding agents. This enables us to study the robustness of these formations via rigidity of the formation graph (an area of graph theory that deals with the characterization of graphs corresponding to formations which are rigid). Generally speaking, a formation, and by extension its underlying graph, is termed rigid if the distance between each pair of agents remains constant over time, normally through a subset of the inter-agent distances being actively maintained at prescribed values. It has been shown that this property is generic, in the sense that the rigidity of almost all formations with the same graph depends only on the topology of its graph and not the actual distances between the agents. This implies that having enough well-distributed control links within the formation will lead to a rigid formation no matter what the actual distances are between agents.

The rigidity of graphs has been extensively studied in the past [3], especially those corresponding to formations in an ambient twodimensional space. However, robustness to multiple agent/link loss is a very new topic. In [17], robustness is defined by introducing the notion of $k$-edge/ $k$-vertex rigidity of a graph: the graph remains rigid after the loss of up to $k-1$ edges/vertices; [17] also derived a collection of general properties of such graphs. In [18], a characterization is derived for $k$-edge rigidity. The author in [13] introduced 
birigid graphs, which are graphs remaining rigid after the loss of one vertex. In a different direction [15] proposes a procedure to restore the rigidity property of a rigid formation, when one of its agents is lost.

The main contribution of this paper is to parallel the path posed in [17], [18] and [15], focusing on the loss of more than one agent. In a related study [10], we have derived some distinct necessary and sufficient conditions for graphs which are $k$-vertex globally rigid, a stronger condition than rigidity. The similar results for rigidity will be provided here as a partial characterization of $k$-vertex rigid graphs, i.e. graph which remain rigid after the loss of up to $k-1$ vertices. As it turns out, for $k \leq 3$ a size independence property holds, i.e. the cost, in terms of extra edges required to secure the $k$-vertex rigidity property as opposed to mere rigidity, is very small and independent of the number of agents in the formation. As is later explained, this low cost property is lost when robustness against the loss of more than 2 agents is sought. This observation reinforces our focus on 3 -vertex rigid graphs with the minimum edge count. A partial characterization of these graphs will be derived, followed by a constructive approach through which one can obtain a 3-vertex graph on arbitrary number of vertices. This characterization enables the formation designer to embed the robustness into a formation prior to any actual mission.

The structure of the paper is as follows. In Section II, some basic definitions are provided. Section III contains the main contribution of the paper and is followed by the concluding remarks in Section IV.

\section{BACKGROUND}

Rigidity theory has been well studied in the literature [3], [16]. In this section, we introduce some basic definitions and properties required for the main contribution. The interested reader may refer to [3] for further details. Throughout this paper we assume that the formation lies in an ambient two-dimensional space and is modeled by a graph whose rigidity implies the rigidity of the formation and all generic formations with the same graph [3].

\section{A. Rigidity and Minimal Rigidity}

As mentioned earlier, a graph is called rigid if the only smooth motions are those corresponding to translation and rotation of the whole formation (see [3] for a precise definition). According to [16], a graph is minimally rigid if it is rigid and removing any one of the edges results in a nonrigid graph (for which any corresponding formation would have the possibility of flexing motions, apart from translation and rotation). There is a celebrated theorem, called Laman's Theorem [6], which gives a fully combinatorial characterization of minimally rigid graphs. In [6], it is also proved that every rigid graph contains a minimally rigid subgraph with the same vertices.

\section{B. Redundant Rigidity}

A graph is said to be redundantly rigid, if it is rigid and after removing any of the edges it still remains rigid. This notion can be generalized to the loss of $k$ edges and/or $k$ vertices. A graph will be termed $k$-edge rigid if after deletion of any set of up to $k-1$ edges, a rigid graph always results. With the same notion a graph is $k$-vertex rigid if after deletion of any set of up to $k-1$ vertices, the resulting graph is still rigid. As mentioned before, we are interested in addressing multiple agent loss. Therefore, in the remainder of the paper we study $k$-vertex rigidity of the underlying graphs. The following theorem and lemma were first proved in [17] and due to their relevance are restated here (we provide the proof of Lemma 2 as it summarizes several observations in [17]):
Theorem 1. If $G=(V, E)$ is a $k$-vertex rigid graph, then each vertex has a degree of at least $k+1$.

Lemma 2. In a $k$-vertex rigid graph $G=(V, E),|V| \geq k+3$ except for $K_{k+2}$, the complete graph on $k+2$ vertices.

Proof: Suppose to obtain a contradiction that $|V| \leq k+2$ and $G$ is not $K_{k+2}$. The $k$-vertex rigidity of $G$ implies $\delta_{G}(v) \geq k+1$ (degree of vertex $v \in V$ ) which implies that $|V|$ cannot be less than $k+2$. However, the only graph with $|V|=k+2$ in which $\delta_{G}(v) \geq k+1$ holds is $K_{k+2}$. This contradiction implies $|V| \geq k+3$.

A graph is called minimally $k$-vertex ( $k$-edge) rigid, if it is $k$-vertex ( $k$-edge) rigid but after removing any one of the edges the resulting graph is no longer $k$-vertex ( $k$-edge) rigid.

In the case of mere rigidity, the definition of minimal rigidity is proved to be equivalent to an alternative statement: a rigid graph is called minimally rigid if it has the minimum number of possible edges among all rigid graphs with the same number of vertices. Unfortunately, for general redundant rigidity ( $k$-edge rigidity or $k$ vertex rigidity), these two notions are no longer equivalent; there are some graphs which are minimally redundantly rigid but the number of edges is not the minimum possible one among such graphs with the same vertex count. This property of redundant rigidity leads us to two different notions: strongly minimal and weakly minimal redundant rigidity [15].

- A $k$-vertex (k-edge) rigid graph is said to be strongly minimal if it has the minimum possible number of edges on a given number of vertices.

- A $k$-vertex (k-edge) rigid graph is said to be weakly minimal if it has more than the minimum possible number of edges on a given number of vertices, but has the property that removing any edge destroys $k$-vertex ( $k$-edge) rigidity.

Later, we will need the characterizing conditions for 2 -vertex rigidity as an special case. The remainder of this section contains the results to characterize strongly minimal 2-vertex rigid graphs [13]. Figure 1, originally from [13], depicts examples of strongly minimal 2-vertex rigid graphs.

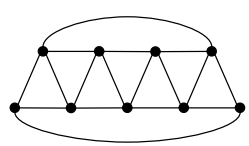

(a)

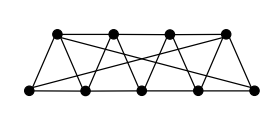

Figure 1. Examples of the 2 possible partitions of the edge set for strongly minimal 2-vertex rigid graphs: (a) the degree 3 vertices are adjacent, (b) the degree 3 vertices are non-adjacent

Lemma 3. If $G=(V, E)$ is a 2-vertex rigid graph on 5 or more vertices, then $|E| \geq 2|V|-1$.

Theorem 4. Let $G=(V, E)$ be a strongly minimal 2-vertex rigid graph on 5 or more vertices. Then $G$ has exactly 3 vertices with degree 3 and the remaining vertices have degree 4 , which implies $|E|=2|V|-1$.

Theorem 5. A graph $G=(V, E)$ is strongly minimal 2-vertex rigid if and only if $G$ has exactly two vertices of degree 3 and there is a partition of the edge set $E$

$$
E=E_{1} \cup E_{2} \cup \ldots \cup E_{k}
$$

such that the graph induced by $E \backslash E_{i}$ is minimally redundantly rigid (i.e. the removal of any edge destroys redundant rigidity) for all $i$, and either 
- $E_{1}$ and $E_{2}$ are the edges incident to the two non-adjacent vertices of degree 3 , respectively, and $E_{i}$ is a single edge for $3 \leq i \leq k$, or

- $E_{1}$ is the union of the edges incident to the two adjacent vertices of degree 3 , and $E_{i}$ is a single edge for $2 \leq i \leq k$.

\section{RESULTS}

In this section we start by studying a sufficient condition for $k$-vertex rigidity (Section III-A). This will enable us to propose formation structures which are robust against the loss of up to $k-1$ agents. Then, from a different perspective, the structure of $k$-vertex rigid graphs is studied and a distinct necessary condition is obtained (Section III-B). We will show the size independence property for $k \leq 3$ and display the optimality of structures which are 3-vertex rigid (highest value of $k$ with size independence property). Therefore, we will some characterization of the special case of 3-vertex rigidity. It will be done by introducing some results on the relation between vertex and edge counts and vertex degrees of these graphs. Starting from $|V|=6$, we will propose a constructive approach which can grow such graphs to arbitrary size via an extension operation.

\section{A. Sufficient Condition for $k$-Vertex Rigidity}

The notion of $k$-connectivity has been well studied in the literature and there are efficient algorithms to check this property on a given graph [11]. The idea here is to find a $(k+j)$-connectivity condition which is sufficient to $k$-vertex rigidity. The motivation comes from [8] where it is shown that in $2 D, 6$-connectivity implies rigidity and 6 is the least possible number for this condition ( $k$-connectivity with $k<6$ is not sufficient for rigidity). The following theorem is derived from a stronger theorem we first proposed in [10].

We can extend this result to the case of $k$-vertex rigidity, as the following theorem shows:

Theorem 6. Assume that $G=(V, E)$ is a $(k+5)$-connected graph. Then $G$ is k-vertex rigid.

The importance of this result is that it shows that the more complicated and awkward-to-verify property of $k$-vertex rigidity can be reduced to a well-known and easier to study property of $k$ connectivity.

\section{B. Necessary Condition for $k$-Vertex Rigidity}

In this subsection we derive a necessary condition similar to what we have derived in [10] but for $k$-vertex rigidity. This result gives a lower bound on the number of edges of such graphs. From [6], [13] we know that the minimum required number of edges for a graph to be rigid and 2-vertex rigid are $|E|=2|V|-3$ and $|E|=2|V|-1$, respectively. This observation leads us to an important question: since in both the above lower-bounds the number of edge is twice the number of vertices $(|V|)$ plus a constant, can we conjecture that for any $k \geq 2$, the edge count of any strongly minimal $k$-vertex rigid graph satisfies the condition $|E|=2|V|+c(k)$ ( $c$ is independent of $|V|)$ ? Unfortunately, the answer is no and, as we show in Theorem 8 , this property is only valid for $k \leq 3$. For such values of $k$ we say that the $k$-vertex rigid graph, has the size independence property, meaning that the number of edges required to obtain a $k$-vertex rigid graph from a rigid graph is independent of the number of vertices $(|V|)$.

Theorem 7 gives a lower bound on the edge count of a $k$-vertex rigid graph. We should mention that the same result is valid for $k$ edge rigid graphs as well.
Theorem 7. In a strongly minimal $k$-vertex rigid graph, with $k \geq 2$, the edge count is under-bounded by the formula $|E| \geq\left\lceil\frac{k+1}{2}|V|\right\rceil+$ $c(k)$, where $c(k)$ is an integer ( $c$ is independent of $|V|$ but depends on $k$ ) and for $k \geq 3$, if the equality holds (i.e. $|E|=\left[\frac{k+2}{2}|V|\right\rceil+c(k)$ ), then $c(k) \geq 0$.

Proof: Assume that $G=(V, E)$ is a strongly minimal $k$-vertex rigid graph of $|V| \geq k+3$ vertices, whose number of vertices is $|E|=a|V|+c(k)$ (according to Theorem 11 in [10] such a graph exists), where $c(k)$ is independent of $|V|$. According to Theorem 1, $\delta_{i} \geq k+1$ holds. Therefore, the average degree in $G$ is $\delta_{a v g} \geq k+1$. On the other hand, $\delta_{a v g}=\frac{2|E|}{|V|}=2 a+\frac{2 c(k)}{|V|}$. Hence, $2 a+\frac{2 c(k)}{|V|} \geq$ $k+1 \Rightarrow k \leq(2 a-1)+\frac{2 c(k)}{|V|}$.

Since the property must hold for graphs of arbitrary size and in particular arbitrarily large $|V|$, assuming $|V|>2(c(k)$, we will have $k \leq 2 a-1$ or $a \geq \frac{k+1}{2}$. Therefore, $|E| \geq\left\lceil\left(\frac{k+1}{2}\right)|V|\right\rceil+c(k)$ holds for $|V|>2 c(k)$.

Now suppose that for some $|V|$ the equality holds (i.e. $|E|=$ $\left.\left\lceil\frac{k+1}{2}|V|\right\rceil+c(k)\right)$. We prove that for such a strongly minimal $k$ vertex globally rigid graph $c(k) \geq 0$ always holds.

First suppose $k$ is odd. Then, we will have $\delta_{\text {avg }}=k+1+\frac{2 c(k)}{|V|} \geq$ $k+1$, which implies $c(k) \geq 0$.

If $k$ is even, then $|E|=\frac{\bar{k}}{2}|V|+\left\lceil\frac{|V|}{2}\right\rceil+c(k)$ which gives $|E|<$ $\frac{k}{2}|V|+c(k)+\frac{|V|}{2}+1$. Therefore, $\delta_{\text {avg }}<k+\frac{2 c(k)}{|V|}+1+\frac{2}{|V|}$ holds. This implies $k+1<k+1+\frac{2 c(k)}{|V|}+\frac{2}{|V|}$ which gives $-1<c(k)$ and so $c(k) \geq 0$ holds.

Theorem 8. The highest value of $k$ which the $k$-vertex rigid graph $G_{k}=(V, E)$ can have the size independence property is $k=3$.

Proof: As is shown in the proof of Theorem 7, the inequality $k \leq 2 a-1$ holds. On the other hand, if $G_{k}$ has the size independence property, then we necessarily must have $a=2$ (as for rigidity and 2 -vertex rigidity $a=2$ ). This implies $k \leq 3$. Therefore $\operatorname{argmax}_{k}\left(G_{k}\right)=3$ holds for size independent graphs $G_{k}$ and the proof is complete.

From this result we can conclude that a formation designer can embed the robustness to the loss of up to two agents into a formation of any agent count, by adding only a fixed set of control links with possible redistribution of some edges to be incident on different vertex pairs. Actually by studying 3-vertex rigid graphs in the next section, we will derive the exact number of required control links to obtain such a robustness level (Lemma 9).

The size independence property is not true when there is robustness to the loss of more than two agents. Table I shows an example of a formation with 100 agents and the required number of control links for any desired level of robustness. As is evident, there is a considerable increase in the required number of control links when we want to obtain robustness to the loss of more than two agents.

\begin{tabular}{|c|c|}
\hline \# of agent losses tolerated & \# of required links \\
\hline \hline 0 & 197 \\
\hline 1 & 199 \\
\hline 2 & 202 \\
\hline 3 & $250+c^{+}$ \\
\hline
\end{tabular}

THE REQUIRED NUMBER OF CONTROL LINKS IN A 100-AGENT FORMATION WITH DIFFERENT ROBUSTNESS PROPERTIES. $c^{+}$IS A POSITIVE INTEGER.

\section{Relations between edge count, vertex count and vertex degrees}

In this subsection, we study 3 -vertex rigidity by presenting some results linking the vertex and edge counts and vertex degrees. The 
first result underbounds the edge count in a 3-vertex rigid graph in terms of the vertex count.

Lemma 9. If $G=(V, E)$ is a 3-vertex rigid graph with $|V| \geq 6$, then $|E| \geq 2|V|+2$.

Proof: First we prove that the inequality $|E| \geq 2|V|+1$ holds. It is then enough to show that there is no 3 -vertex rigid graphs on $|E|=2|V|+1$ edges.

For the inequality, to obtain a contradiction, suppose $G$ is a 3vertex rigid graph and has $|E| \leq 2|V|$ edges. In this case the average vertex degree is at most 4 . Since all vertex degrees must be at least 4 , this implies that the degree of each vertex is precisely 4 , so that $|E|=2|V|$.

Now consider $v_{1}, v_{2} \in V$ of degree 4 which are not adjacent to each other (and always exist since $|V| \geq 6$ ), and remove them from $G$ to produce a graph $G^{\prime}=\left(V^{\prime}, E^{\prime}\right)$. Then $\left|E^{\prime}\right|=2|V|-8=$ $2\left|V^{\prime}\right|-4$. Obviously $G^{\prime}$ is not rigid, which contradicts the fact that $G$ is 3 -vertex rigid.

To prove that $|E|=2|V|+1$ is impossible, suppose that the equality holds and set $n=|V|$. The average vertex degree in $G$ is $\frac{\sum \delta_{i}}{n}=\frac{2|E|}{n}=\frac{4 n+2}{n}=4+\frac{2}{n}>4$. Therefore, there is at least one vertex with degree of more than 4 . Now suppose $v_{1}, v_{2} \in V$ with degrees $k_{1}$ and $k_{2}$, respectively. Observe that $G-v_{1}-v_{2}$, obtained by removing $v_{1}, v_{2}$ and all their incident edges from $G$, has $n-2$ vertices and at most $|E|=2 n+1-\left(k_{1}+k_{2}-1\right)=2(n-2)-\left(k_{1}+k_{2}-6\right)$ edges (the bound being achieved when $v_{1}, v_{2}$ are neighbors). Since $G-v_{1}-v_{2}$ is rigid, $|E| \geq 2(n-2)-3$ must hold. So $k_{1}+k_{2}-6 \leq 3$ or $k_{1}+k_{2} \leq 9$. By considering $k_{1} \geq 4, k_{2} \geq 4$ we conclude that $4 \leq k_{1} \leq 5$ and $4 \leq k_{2} \leq 5$. Finally, if there are $m$ vertices of degree 5 , we have $5 m+4(n-m)=2(2 n+1)$, which gives $m=2$. This means that such a graph (if it exists) should have exactly two vertices of degree 5 which are adjacent and the others with degree 4 .

Now we prove that such a graph cannot be 3 -vertex rigid. First consider the case that $n=6$. In this case all vertices of degree 4 are connected to vertices of degree 5 (figure 2). It is obvious that by removing vertices with degree 5 , the resulting graph is not rigid. Now consider $n \geq 7$. In this case there are at least one pair $\left(v_{1}, v_{2}\right)$ with degrees 4,5 which are not adjacent. $G-v_{1}-v_{2}$ has $2 n+1-5-4=$ $2 n-8=2(n-2)-4$ edges which contradicts the fact that it is rigid. This completes the proof.

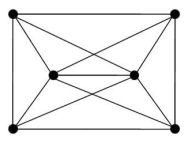

Figure 2. A graph with two 5-degree and four 4-degree vertices.

Figure 3 shows an example of a strongly minimal 3-vertex rigid graph with $|E|=2|V|+2$ on 6 vertices. Later in Section III-D, we will show that this class of graphs is an infinite set, by introducing an extension operation which can grow a strongly minimal 3 -vertex rigid graph by one vertex. In the following theorem an in anticipation of the demonstration of existence of such graphs for arbitrary $|V| \geq 6$, we present a condition on the vertex degrees of these graphs.

Theorem 10. There exists a strongly minimal 3-vertex rigid graph $G=(V, E)$ with $|E|=2|V|+2$ for any $|V| \geq 6$. In $G$, there are exactly 4 vertices with degree of 5 which are all adjacent (forming a $K_{4}$ subgraph) and all other vertices have degree of 4 .

Proof: This graph has an average degree of $4+\frac{4}{n}$ (taking $|V|=$ $n$ ). Therefore, there is at least one vertex with degree of more than 4. Now suppose $v_{1}, v_{2} \in V$ with degrees $k_{1}, k_{2}$, respectively. Then, $G-v_{1}-v_{2}$ has $n-2$ vertices and at most $\left|E\left(G-v_{1}-v_{2}\right)\right|=$
$2 n+2-\left(k_{1}+k_{2}-1\right)=2(n-2)-\left(k_{1}+k_{2}-7\right)$ edges (when $v_{1}, v_{2}$ are neighbors). Since $G-v_{1}-v_{2}$ is rigid, $\left|E\left(G-v_{1}-v_{2}\right)\right| \geq$ $2(n-2)-3$ holds. Hence, $k_{1}+k_{2}-7 \leq 3$ or $k_{1}+k_{2} \leq 10$. By considering $k_{1} \geq 4, k_{2} \geq 4$ we conclude that $4 \leq k_{1} \leq 6$ and $4 \leq k_{2} \leq 6$.

Finally, if there are $m$ vertices of degree 6 and $t$ vertices of degree 5 , we have $6 m+5 t+4(n-m-t)=2(2 n+2)$, which gives $2 m+t=4$. There are 3 possible cases:

a. $m=1, t=2$ : in this case if we remove the 6 -degree vertex in addition to a 5-degree one, the number of edges will be at most $\left|E\left(G-v_{1}-v_{2}\right)\right|=2 n+2-(6+5-1)=2 n-8=2(n-$ 2) -4 which contradicts the fact that $G-v_{1}-v_{2}$ is rigid and $\left|E\left(G-v_{1}-v_{2}\right)\right| \geq 2(n-2)-3$. Therefore, this case cannot occur.

b. $m=2, t=0$ : with the same argument as the case $\mathbf{a}$, by removing two vertices with degree of 6 we have $\left|E\left(G-v_{1}-v_{2}\right)\right|=$ $2 n+2-(6+6-1)=2 n-9=2(n-2)-9$ and it is obvious that the resulting graph is not rigid. Hence again, this case cannot occur.

c. $m=0, t=4$ : The proof of this case is trivial. The only important condition is that all 5-degree vertices should be adjacent. Otherwise, removing any 2 of them results in a non-rigid graph $\left(\mid E\left(G-v_{1}-v_{2} \mid=2(n-2)-4<2(n-2)-3\right)\right.$. Figure 3 shows such a graph with 6 vertices.

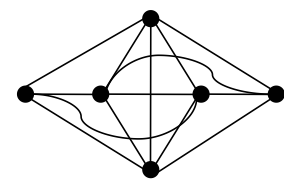

Figure 3. Strongly Minimal 3-vertex rigid graph with $|V|=6$.

Remark 11. Observe that the condition provided by theorem 10 is not sufficient to ensure strongly minimal 3-vertex rigidity. As a counter example, consider the graph $G$ shown in Figure 4 (left side). It is easy to observe that this graph satisfies Theorem 10. However, as shown in the right side of the figure, removing vertices shown by unfilled circles results in a non-rigid graph. Therefore, $G$ is not 3-vertex rigid.

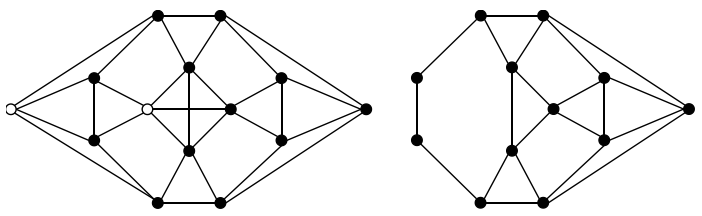

Figure 4. No 3-vertex rigid graph which satisfies Theorem 10

\section{Growing strongly minimal 3-vertex rigid graphs}

In this section we will show an infinite class of strongly minimal 3vertex rigid graphs. The approach is to propose an extension operation which can be applied in any strongly minimal 3 -vertex rigid graph and increases its vertex count by one. This operation is a special case of $X$ replacement operation [15] used before for extending weakly minimal 2 -vertex rigid graphs - we call this new one 4-5 X-Replacement, for reasons which are about to become apparent. Suppose that the original graph is $G=(V, E)$. Choose two edges $e_{1}=\{a, b\}$ and $e_{2}=\{c, d\}$ and $e_{1}, e_{2} \in E$ so that $a, c$ have degree 4 and are non-adjacent and $b, d$ have degree 5 (that such a choice is in fact possible is proved below). Remove $e_{1}, e_{2}$ and add a new vertex called $z$. Connect $z$ to $a, b, c, d$. In 4-5X-Replacement operation the degree of the original vertices remains the same and a new vertex of degree 4 is added 
to $G$. Therefore, the new graph satisfies the conditions of the above theorems.

It remains to prove that this operation preserves strongly minimal 3 -vertex rigidity. First we provide a lemma which guarantees that there are always appropriate edge choices for 4-5 X-replacement operation.

Lemma 12. If $G=(V, E)$ is a strongly minimal 3-vertex rigid graph, there are always two vertices, a, c say, with degree of 4 which are not adjacent, yet are connected to two different vertices, $b$ and $d$ say, with degree of 5.

Proof: Since the vertices of degree 4 which are connected to the core (vertices of degree 5) can only be adjacent to at most 3 other vertices of the same degree 5 , if the number of vertices of degree 4 is more than 4, there are at least two of them which are not adjacent. Therefore, the theorem is proved for $|V|>8$. Since $|V| \geq 6$ holds in general, we need to prove the lemma for $|V|=6,7$ and 8 . From Figure 3 it is evident that in the only strongly minimal 3 -vertex rigid graph with 6 vertices, the only two 4-degree vertices are not adjacent. Finally, it is trivial to see that (up to isomorphism) the graphs in Figure 5 are the only possible strongly minimal 3-vertex rigid graphs of size 7 and 8 respectively.

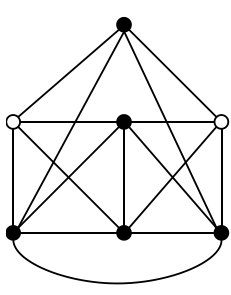

a

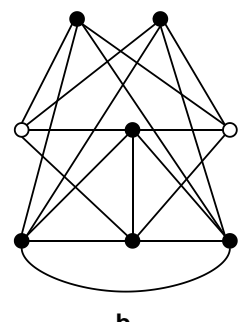

b
Figure 5. Strongly minimal 3-vertex rigid graphs of size 7 (a) and 8 (b) Vertices depicted by unfilled circles are 4-degree but not adjacent.

Theorem 13. Suppose $G=(V, E)$ is a strongly minimal 3-vertex rigid graph. After applying the 4-5 X-Replacement operation on $G$, the resulting graph $G^{\prime}$ is strongly minimal 3-vertex rigid on $|V|+1$ vertices.

Proof: In this proof we show that by removing any pair of vertices from the graph $G^{\prime}$ after 4-5 X-replacement, the resulting graph is still rigid. Suppose $(a, b)$ and $(c, d)$ are two candidate edges to be removed from $G$ in 4-5X-replacement, with $\delta_{a}=\delta_{c}=4$ and $\delta_{b}=\delta_{d}=5$. The new vertex to be connected to all of $a, b, c, d$ is $e$ (Figure 6).
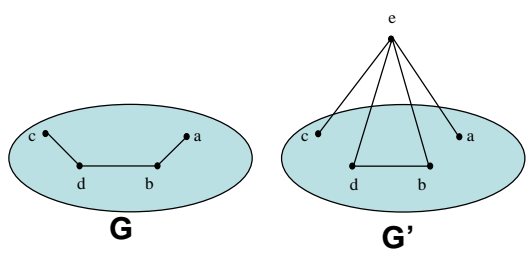

Figure 6. 4-5 X-replacement operation. $b, d$ have degree 5 while $a, c$ have degree 4.

There are 6 distinct cases based on different choices for the vertex pair to be removed from $G^{\prime}$. For further reference we call these vertices $x$ and $y$.

a. $x, y \in V \backslash\{a, b, c, d\}$ : We know that $G-x-y$ is rigid. In this case, none of the edges connecting $e$ to one of $\{a, b, c, d\}$ is removed. Therefore, one can interpret $G^{\prime}-x-y$ as an extension of $G-x-y$ by an ordinary $X$-replacement operation. This has been proved to preserve rigidity of $G^{\prime}-x-y$ [15]. Hence, in this case the result of the operation is still a rigid graph.

b. $x \in\{a, b\}$ (or $x \in\{c, d\}$ which are the same by the symmetry property) and $y \in V \backslash\{a, b, c, d\}$ : Without loss of generality suppose that for this case $x=a$ (Figure 7). Therefore, $G^{\prime}-x-y$ can be obtained from $G-x-y$ by a standard edge splitting operation which preserves rigidity [16]. Since $G-x-y$ is rigid, $G^{\prime}-x-y$ is also rigid.
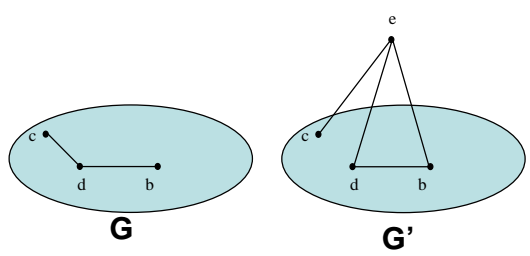

Figure 7. Removing $x=a$ and $y \in V \backslash\{a, b, c, d\}$ from $G$ and $G^{\prime}$. The operation reduces to the edge splitting operation on $(c, d)$ edge.

c. $x \in\{a, b\}$ and $y \in\{c, d\}$ (one vertex from each edge). Again suppose that -without loss of generality- $x=a$ and $y=c$ (Figure 8). One can simply observe that $G^{\prime}-x-y$ can be produced by applying a vertex addition to $G^{\prime}-x-y$ which preserves rigidity when applied to a rigid graph [16]. Therefore, $G^{\prime}-x-y$ is rigid.

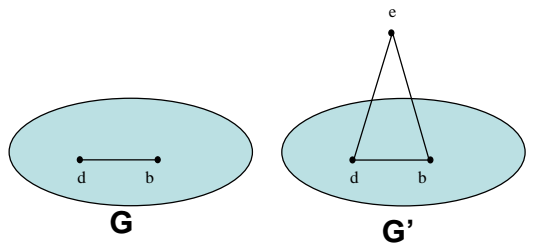

Figure 8. Removing $x=a$ and $y=c$. The operation reduces to vertex addition operation in which $e$ is connected to $b, d$ by 2 edges.

d. $x=a$ and $y=b$ (both are the extremes of one edge): Without loss of generality suppose that $x=a$ and $y=b$ (Figure 9). After removing vertex $b$, the resulting graph $\left(G^{\prime}=G-b\right)$ is still 2-vertex rigid and has $\left|E^{\prime}\right|=2|V|+2-5=2\left|V^{\prime}\right|-1$ edges. According to theorem 4 and 5 , it is strongly minimal 2 -vertex rigid with exactly two vertices of degree 3 (which had been 4-degree vertices connected to $b$ before it was removed). Since vertex $a$ is one of the 3-degree vertices in this graph, according to theorem 5, removing it and all of its incident edges ensures that the resulting graph is redundantly rigid. Therefore, $G-x-y-(c, d)$ is still rigid. One can easily conclude that adding $e$ with two edges to this graph, which forms $G^{\prime}-x-y$, is a case of vertex addition extension of a rigid graph and therefore $G^{\prime}-x-y$ is rigid.

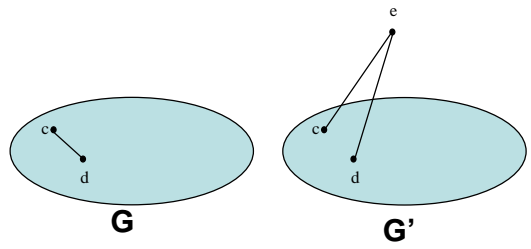

Figure 9. Removing $x=a$ and $y=b$.

e. $x=e$. In this case if $y \in\{a, b, c, d\}$, we can observe that the difference between $G-y$ and $G^{\prime}-x-y$ is that $G-y$ has an extra 
edge $(c, d)$. Since $G-y$ is 2 -vertex rigid, we can conclude that it is 2-edge rigid [18]. Therefore, by removing one edge like $(c, d)$ from it, the result $\left(G^{\prime}-x-y\right)$ is still rigid (Figure 10).

If $y \notin(a, b, c, d\}$, the difference between $G^{\prime}-x-y$ and $G-y$ is in two edges $(c, d)$ and $(a, b)$ (Figure 11). From theorem 5 we know that $G-y-(c, d)$ is minimally redundantly rigid (since $E_{i}=(c, d)$ in one possible partition of $G-y)$. Therefore, $G-y-(c, d)-(a, b)$ is rigid. By looking at Figure 11 one can easily realize that $G-y-$ $(c, d)-(a, b)$ is $G^{\prime}-y-x$. Hence, $G^{\prime}-y-x$ is rigid.
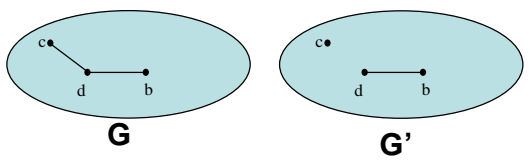

Figure 10. Removing $x=e$ and $y=a$.

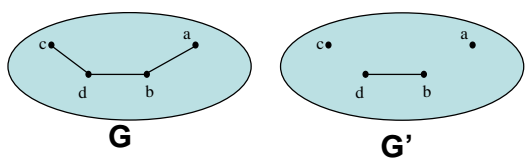

Figure 11. Removing $x=e$ and $y \in G \backslash\{a, b, c, d\}$

This completes the proof of Theorem 13 .

\section{CONCLusions}

In this paper we studied the robustness of information architectures to control the formation of autonomous agents. As these agents sometimes operate in hazardous environments like battle-fields, they are prone to multiple agent and/or link loss. Due to the higher severity of agent loss than link loss, the main contribution of this paper is to propose information architectures for multi-agent formations, which are robust against the loss of multiple agents, in the sense of retaining rigidity of the formation when such loss occurs. By adopting the notion of $k$-vertex rigid graphs (corresponding to formations which are tolerant to the loss of up to $k-1$ agents, we derived some distinct necessary and sufficient conditions. We also established the size independence property for $k \leq 3$, i.e. the cost, in terms of extra edges required to secure the $k$-vertex rigidity property as opposed to mere rigidity, is very small and independent of the number of agents in the formation. As explained before, this low cost property is lost when robustness against the loss of more than two agents is sought. This observation motivated the detailed study of 3-vertex rigid graphs with the minimum edge count. A partial characterization of these graphs was derived, followed by a constructive approach through which one can obtain a 3-vertex rigid graph on arbitrary number of vertices. This characterization enables the formation to be designed with the robustness against the loss of up to two agents prior to any actual mission, a proactive approach.

The main assumption in this paper was that the control operation is cooperative in the sense that both agents at the ends of a control link, cooperate to maintain the desired distance. This enabled us to model the formation with an undirected graph. However, there are other schemes in which only one of the agents is responsible to maintain a distance. These problems and the robustness of such formations can be studied via the definition of persistent formations [2] as an extension of this work.

Finally, although we provided some necessary and sufficient conditions on $k$-vertex rigid formations, it is still an open problem to fully characterize such architectures. This is one possibility for future work.

\section{REFERENCES}

[1] B. Anderson, B. Fidan, C. Yu, and D. Walle. UAV formation control: Theory and application. Recent Advances in Learning and Control, pages $15-33,2008$.

[2] B. Anderson, C. Yu, B. Fidan, and J. Hendrickx. Rigid graph control architectures for autonomous formations. Control Systems Magazine, IEEE, 28(6):48-63, 2008.

[3] J.E. Graver, B. Servatius, and H. Servatius. Combinatorial rigidity. American Mathematical Society, 1993.

[4] Daniel L. Haulman. U.s. unmanned aerial vehicles in combat. Technical report, Air Force Historical Research Agency, 2003.

[5] T. Kopfstedt, M. Mukai, M. Fujita, and C. Ament. Control of Formations of UAVs for Surveillance and Reconnaissance Missions. In 17th IFAC World Congress, COEX, South Korea, pages 6-11, 2008.

[6] G. Laman. On graphs and rigidity of plane skeletal structures. Journal of Engineering Mathematics, 4:331-340, 1970.

[7] Jay Levine. Nasa j-ucas $x-45 a$ represents an evolutionary step in technology and a look at future aviation. The X-press, Dryden Flight Research Center, 46(7), 2004.

[8] L. Lovasz and Y. Yemini. On generic rigidity in the plane. SIAM Journal on Algebraic and Discrete Methods, 3:91, 1982.

[9] R.K. Mehra, J.D. Boskovic, and S.M. Li. Autonomous formation flying of multiple UCAVs under communication failure. In Position Location and Navigation Symposium, IEEE 2000, pages 371-378. IEEE, 2000.

[10] S. Alireza Motevallian, Changbin Yu, and Brian D. O. Anderson. Robustness to the loss of multiple nodes in the localizability of sensor networks. To be published in IFAC WC 2011, August 2011.

[11] H. Nagamochi and T. Ibaraki. Algorithmic aspects of graph connectivity. Cambridge University Press New York, NY, USA, 2008.

[12] T. Nisser and C. Westin. Human factors challenges in unmanned aerial vehicles (uavs): A literature review. Lund University School of Aviation, Tech. Rep. TFHS, 5:1, 2006.

[13] B. Servatius. Birigidity in the plane. SIAM Journal on Discrete Mathematics, 2(4):582-599, 1989.

[14] R.S. Smith and F.Y. Hadaegh. Control of deep-space formation-iňĆying spacecraft: relative sensing and switched information. AIAA Journal of Guidance, Control, and Dynamics, 28(1):106-114, 2005.

[15] T.H. Summers, C. Yu, and B. Anderson. Addressing agent loss in vehicle formations and sensor networks. International Journal of Robust and Nonlinear Control, 19(15):1673-1696, 2009.

[16] T.S. Tay and W. Whiteley. Generating isostatic frameworks. Structural Topology 1985 núm 11, 1985.

[17] C. Yu and B. D. O. Anderson. Development of redundant rigidity theory for formation control. International Journal of Robust and Nonlinear Control, 19(13):1427-1446, 2009.

[18] C. Yu, S. Dusgupta, and B. D. O. Anderson. Network localizability with link or node losses. In Proceedings of the 49th IEEE Conference on Decision and Control, pages 402-407, 2010. 